patients; the thresholds of benefit that would produce adherence were also assessed.

Methods Four methods of displaying information about the benefits of maintenance therapy in remission were explained to UC patients in remission, during face to face structured interviews. These were largely conventional numerical approaches: relative risk reduction $[R R]$, absolute risk reduction $[A R]$, number needed to treat [NNT]. The fourth was an optical representation via Cates plot [CP]). Patients understanding and preference for each approach were recorded. Patients were asked to state the minimum thresholds required to adhere to a hypothetical medication (with 5-ASA like properties) for the benefits of relapse and cancer reduction respectively. Thresholds were determined for each method of display.

Results Of 50 participants (mean age 50 years; 58\% male) 48\% preferred data presentation by RR over CP (28\%), AR (20\%) and NNT (4\%). 94\% found RR easy to understand, better than CP (74\%), AR (88\%) or NNT (48\%). Thresholds required for adherence also differed between methods. For bowel cancer prevention, $94 \%$ indicated adherence for benefit levels of $61 \%$ RR or lower but only $57 \%$ would adhere when presented with the corresponding CP $(p<0.001)$. For relapse prevention, $78 \%$ of patients chose a threshold of $40 \%$ or lower but only $43 \%$ chose the corresponding CP $(p<0.001)$. When presented with RR, adherence minimum thresholds equivalent or lower to the actual 5-ASA benefits were applied by $98 \%$ of patients for cancer reduction and $78 \%$ for flare reduction. Conclusion Ulcerative colitis patients prefer RR and CP as methods to display medication benefit. NNT is poorly understood and unpopular. Patients apply significantly higher thresholds for adherence when presented with CP in comparison to RR. Presented with information in this way, most patients would choose to adhere to 5-ASA medication when offered the actual benefit profile. Reduction of cancer risk may be a stronger motivator than maintenance of remission. Interventions to improve 5-ASA adherence should use RR and convey benefits for cancer and flare prevention.

Competing interests C Selinger: Grant/Research Support from: Shire, Ferring, Nycomed, Y Kinjo: None declared, J McLaughlin: None declared, A Robinson: None declared, R Leong: Grant/Research Support from: Shire, Ferring, Nycomed.

\section{PM0-242 THE IBD-CONTROL QUESTIONNAIRE: DEVELOPMENT AND PSYCHOMETRIC VALIDATION OF A TOOL FOR CAPTURING DISEASE CONTROL FROM THE PATIENT PERSPECTIVE FOR USE IN ROUTINE CARE}

doi:10.1136/gutjnl-2012-302514b.242

${ }^{1} \mathrm{C}$ Ormerod, ${ }^{* 1} \mathrm{D}$ Shackcloth, ${ }^{1} \mathrm{M}$ Harrison, ${ }^{1} \mathrm{E}$ Brown, ${ }^{2} \mathrm{~K}$ Bodger. ${ }^{1}$ Digestive Diseases Centre, Aintree University Hospital, Liverpool, UK; ${ }^{2}$ Department of Gastroenterology, Institute of Translational Medicine, University of Liverpool, Liverpool, UK

Introduction Although a range of disease activity measures and $\mathrm{QoL}$ questionnaires is available for IBD, none has found a place in routine clinical practice. This project aimed to develop a tool for capturing disease control from the patient's perspective with measurement properties appropriate for routine clinical practice.

Methods Phase I: Systematic review of existing PROMS, patient focus groups and a steering group to define domains and items for the "IBD-Control". Instrument comprises 13 questions items plus a visual analogue scale (VAS, 0-100) for overall control. Phase II: Prospective validation, patient completion of IBD-Control, QoL questionnaire (UK-IBD-Q), EuroQol (EO5D), Hospital Anxiety \& Depression Score (HADS); clinician assessment (blinded to questionnaire) recording disease activity (Harvey Bradshaw Index, HBI; or Simple Clinical Colitis Activity Index, SCCAI), global clinician assessment (remission; mild; moderate; severe), Montreal Classification, treatment history. Ongoing longitudinal survey (serial questionnaires).
Results 194/200 returned baseline surveys (CD, $n=107$; UC, $n=87$ ). Study population (CD, UC): Age (mean): 41; 48 yrs. Disease duration (mean): 10.5; 10.7 yrs. Prev. Surgery (\%): 50\%; $3.4 \%$. Immunosuppressants (\%): 49.5\%; $27.6 \%$. Biologics (\%): $22.4 \%$; 8.0\%. Disease activity (mean [SD] HBI; SCCAI): 5 [5]; 4 [3]. Measurement properties of IBD-Control: Completion time (mean [SD]): 1 min $15 \mathrm{~s}$ [25s]; Internal consistency: Cronbach's $\alpha$ for all 13 items: 0.838; for sub-group of 8 questions (IBD-Control-8): 0.841 . Strong correlation between IBD-Control-8 sub-score and IBDControl-VAS $(\mathrm{r}=0.79)$. Test-retest reliability for stable patients (Baseline vs 2 week repeat, no change): IBD-Control-8, 15.8 vs 15.6; $\mathrm{p}=0.73$; IBD-Control-VAS, 65.5 vs 68.0, $\mathrm{p}=0.33$. Validity: Moderateto-strong correlations between IBD-Control-8 subscore and IBDControl-VAS vs disease activity, UK-IBD-O and global health state (utility) with $r$ values 0.56 to 0.84 . Discriminant validity (mean scores for remission, mild, moderate, severe): ANOVA $\mathrm{p}<0.01$. Sensitivity to change: (analysis of first 53 follow-ups): No significant changes for stable patients; moderate-to-large responsiveness statistics for IBD-Control-8 and IBD-Control-VAS: (Effect sizes: 0.4-1.6).

Conclusion The IBD-Control shows promise as a rapid ( $<2 \mathrm{~min})$, reliable, valid and sensitive instrument for measuring overall disease control from the patients perspective. Unlike existing PROMS, its ease-of-use and generic applicability make it a candidate for use in routine practice as a decision-support tool for patients and clinicians.

Competing interests C Ormerod: None declared, D Shackcloth: Grant/Research Support from: Abbott Laboratories Ltd, M Harrison: None declared, E Brown: None declared, K Bodger: Grant/Research Support from: Abbott Laboratories Ltd.

\section{PMO-243 FAECAL CALPROTECTIN ANALYSIS: DOES THE METHOD MATTER?}

doi:10.1136/gutjnl-2012-302514b.243

${ }^{1} \mathrm{C}$ Tomkins, ${ }^{* 2} \mathrm{Z}$ Zeino, ${ }^{2} \mathrm{C}$ Nwokolo, ${ }^{1} \mathrm{~S}$ C Smith, ${ }^{2} \mathrm{R}$ Arasaradnam. ${ }^{1}$ Biochemistry, University Hospitals of Coventry and Warwickshire, Coventry, UK; ${ }^{2}$ Gastroenterology, University Hospitals of Coventry and Warwickshire, Coventry, UK

Introduction Faecal calprotectin (FC) is a sensitive marker of intestinal inflammation and is useful to help distinguish between organic and non-organic (functional) disease. The increasing popularity of this test, with various analytical methods available, potentially leads to confusion in interpreting results. The aim of this study was to technically evaluate FC measured by different ELISA methods in secondary/tertiary care.

Methods 62 stool samples were collected from sequential outpatients presenting with chronic diarrhoea. All participants had a colonoscopy with biopsy, to which FC results were compared. FC was measured by ELISA assays: Immundiagnostik PhiCal (version 1) and Buhlmann EK-CAL. A subset were also measured by PhiCal (version 2). Stool was weighed and extracted, and ELISAs performed manually.

Results 38 patients with IBD/other organic bowel disease (mean 36 yrs, range 15-49) and 24 patients with IBS (mean 36 yrs, range $20-48$ ) were sampled. Sensitivity and specificity for active IBD vs IBS using manufacturers' cut-offs of $50 \mu \mathrm{g} / \mathrm{g}$ were: Buhlmann EKCAL $86 \%$ (95\% CI 42 to 99 ) and 60\% (95\% CI 33\% to $83 \%$ ), PPV $50 \%$ ( $95 \%$ CI $22 \%$ to $78 \%$ ), NPV $90 \%$ ( $95 \%$ CI $54 \%$ to $99 \%$ ); PhiCal1 $78 \%$ (95\% CI $40 \%$ to $96 \%$ ) and $92 \%$ (95\% CI $60 \%$ to $100 \%$ ), PPV $88 \%$ (95\% CI $47 \%$ to $99 \%$ ) and NPV $86 \%$ (95\% CI $56 \%$ to $97 \%$ ). Correlation across full range of results were PhiCall vs EK-CAL, $\mathrm{R}^{2}=0.45 ;$ PhiCal2 vs PhiCal1, $\mathrm{R}^{2}=0.54$. However for results $<100 \mu \mathrm{g} / \mathrm{g}$ by PhiCal1, correlations improved that is, $R^{2}=0.64$ and $\mathrm{R}^{2}=0.83$ respectively. Intra-batch imprecision of the whole process, 